This item was submitted to Loughborough's Research Repository by the author.

Items in Figshare are protected by copyright, with all rights reserved, unless otherwise indicated.

\title{
Sampling and characterisation of volatile organic compound profiles in human saliva using a polydimethylsiloxane coupon placed within the oral cavity
}

\section{PLEASE CITE THE PUBLISHED VERSION}

http://dx.doi.org/10.1039/C2AN35432B

\section{PUBLISHER}

RSC Publishing (@ H.J. Martin, S. Riazanskaia and C.L.P. Thomas)

VERSION

AM (Accepted Manuscript)

\section{LICENCE}

CC BY-NC-ND 4.0

\section{REPOSITORY RECORD}

Martin, Helen J., Svetlana Riazanskaia, and C.L. Paul Thomas. 2019. "Sampling and Characterisation of Volatile Organic Compound Profiles in Human Saliva Using a Polydimethylsiloxane Coupon Placed Within the Oral Cavity". figshare. https://hdl.handle.net/2134/9894. 
This item was submitted to Loughborough's Institutional Repository (https://dspace.lboro.ac.uk/) by the author and is made available under the following Creative Commons Licence conditions.

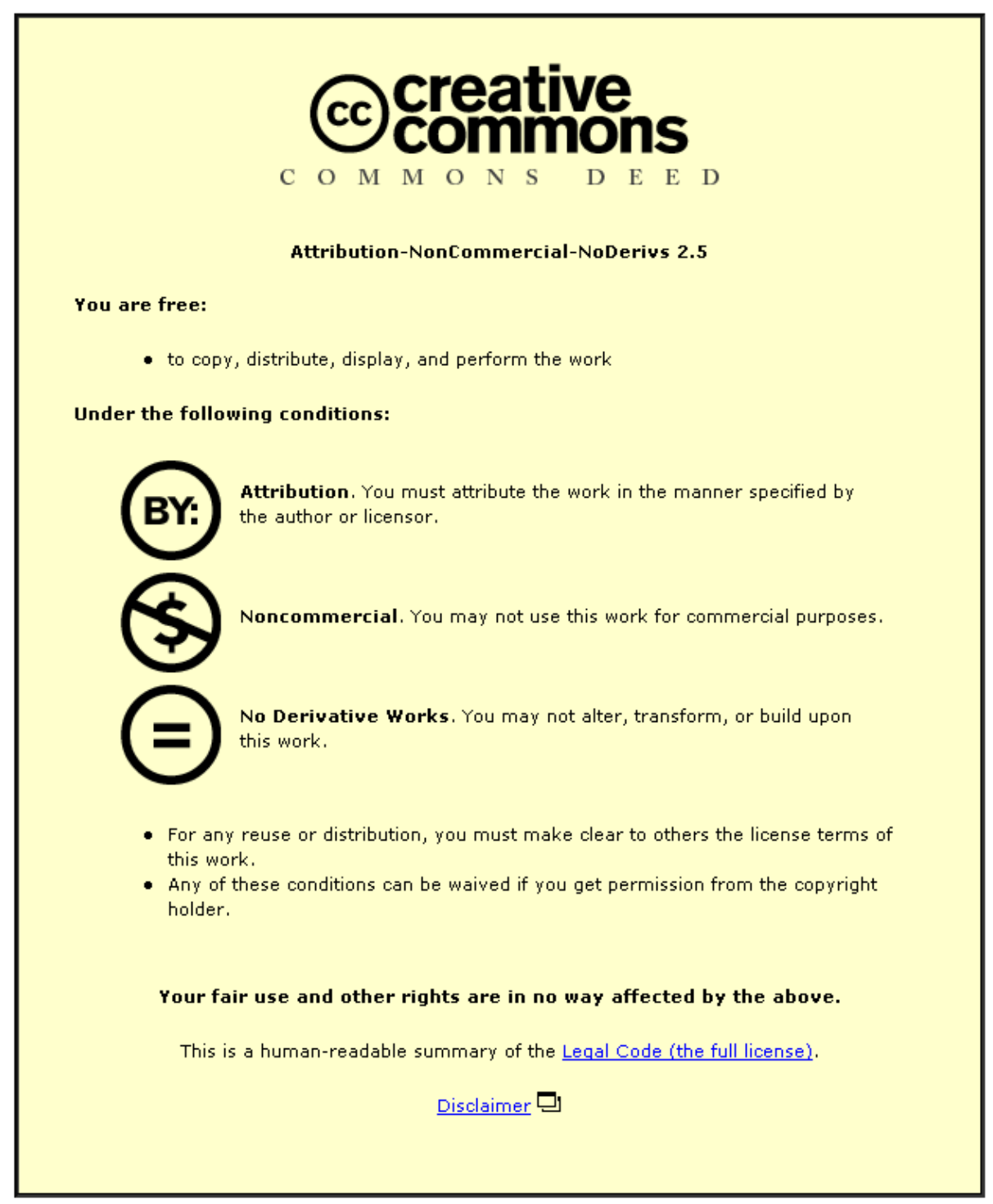

For the full text of this licence, please go to: http://creativecommons.org/licenses/by-nc-nd/2.5/ 


\title{
SAMPLING AND CHARACTERISATION OF VOLATILE ORGANIC COMPOUND PROFILES IN HUMAN SALIVA USING A POLYDIMETHYLSILOXANE COUPON PLACED WITHIN THE ORAL CAVITY.
}

Helen J Martin ${ }^{1}$, Svetlana Riazanskaia,2, ac. L. Paul Thomas ${ }^{1}$.

Centre for Analytical Science, Department of Chemistry, Loughborough University, Loughborough, Leicestershire. LE11 3TU. UK

Unilever R\&D, Port Sunlight, Quarry Road East, Bebington, Wirral, UK. .

\begin{abstract}
Evaluation of published methods reveals that existing methods for saliva sampling do not address the physical chemical attributes of volatile organic compounds (VOC). This study describes and presents evidence for adopting in-situ sampling of salivary VOC directly from the oral cavity using a polydimethylsiloxane (PDMS) based sampler. In-vitro studies indicated that the vapour pressure of analytes was a factor in both the recovery of analytes, and in the precision of the recovery. The highest recoveries were observed for VOC with the lowest vapour pressures, for example 5-nonanol (vapour pressure $\left(P_{v}\right)=14 \mathrm{PA}$ ) recoveries were approximately 20-times greater than those observed for octane $\left(P_{v}=1726 \mathrm{PA}\right)$. Similarly, relative standard deviations reduced with vapour pressure, with the RSD for 5-nonanol responses observed to be $2.7 \%$ to compared to RSD $=26 \%$ for octane. Evaluation of VOC recovered from 6 in-vivo samples indicated that VOC concentrations in saliva may follow lognormal distributions; log-normal RSDs falling between $4.4 \%$ to $18.2 \%$ across the range of volatilities encountered. Increasing sampling time from 1 to 30 minutes indicated that the recovery of VOC into the sampler was effected by interaction between different physical chemical properties and biogenic flux. A sampling time of 10 min was found to offer an acceptable compromise that enabled a representative sample to be acquired for the widest range of observed VOC behaviours with the sampler. The potential to 'tune' the sampling protocol for targeted analysis based on these factors was also noted.
\end{abstract}

Comparison with passive drool saliva collection revealed up to $10^{5}$ enhancment with reduced variability compared to drooled samples. This approach to in-situ saliva sampling appears to have significant analytical utility for studying volatile signatures in humans.

\section{KEYWORDS:}

Gas Chromatography, Mass Spectrometry, Human Saliva, Volatile Organic Compounds, Solid Phase Extraction, Thermal Desorption.

\footnotetext{
a Corresponding Author,c.I.p.thomas@lboro.ac.uk
} 


\section{INTRODUCTION}

Human saliva is a highly variable and individualised biological fluid blended from: the secretions of the salivary glands; gingival crevicular fluid; bacteria and their metabolites; epithelial cells; and food debris, in various stages of decomposition [1]. The composition of whole saliva varies with circadian rhythm as well as in response to physiological and pathological factors such as taste and smell stimuli as well as metabolic influences associated with age; menstrual cycle; physical exercise; and psycho-emotional state. The use of prescription pharmaceuticals and or narcotic substances also effects saliva [2]. Exogenous VOC insults will also show-up in the chemical profile of saliva [3 and 4]. Finally the state of hydration of the individual needs to be included in this list.

The presence of a compound in saliva is determined by its chemical functionality and structural characteristics. Lipophillic and neutral molecules pass from blood to saliva more efficiently than hydrophilic and ionised molecules [5]. The high water level and low protein content of saliva means that strongly protein-bound compounds are unlikely to be present in this matrix [6 and 7].

Saliva sampling and analysis have been used to: monitor toluene exposure and other hazardous VOC in a polymer processing workforce [3 and 4]; measure 3-methoxy-4-hydroxyphenylglycol as a marker of anxiety [8]; diagnose gout from elevated levels of uric acid [9 ]; provide a rapid primary diagnosis of renal disease through uric acid and p-aminohippuric acid determination [10], characterise pharmokinetic profiles of dehydroepiandrosterone and cortisol following corticosteroid administration [11]; indicate cannabis use by measuring $\Delta$ 9-tetrahydrocannabinol [12] and cocaine administration through determination of cocaine, anhydroecgonine methylester, ecgonine methylester and cocaethylene [13]; and to study dietary factors such as fatty acid [14], and caffeine [15 and 16]. Saliva characterisation has also been proposed in relation to systemic diseases such as cystic fibrosis, multiple sclerosis, diabetes mellitus, alcoholic liver sclerosis, acquired human immunodeficiency syndrome and kidney dysfunction [17], as well as its application for qualitative toxicological screening [5].

Quantitative analysis of saliva specimens has indicated meaningful correlations between unbound plasmatic and salivary levels of analytes [7, 8 and 17]. (Note that VOC present in 
plasma as bound complexes are less correlated to salivary levels.) The non-invasive nature and ready accessibility of the sample suggests that saliva may be an attractive alternative to blood and urine for profiling and screening, human and animal subjects, for biological markers.

Protocols for collecting saliva samples include bespoke methods that collect the specific excretions from individual salivary glands; enabling comparison between gland secretions. However, such approaches require high-levels of specialist training to enable reliable sampling. The most common approach, for it is a practical and straightforward procedure, is to collect whole saliva. Even so, this approach requires carefully framed and rigorously conducted protocols to ensure consistency between samples and subjects. Whole saliva can be induced using mastication or citric acid, known as a stimulated method. Alternatively participants may be asked to spit into a vial or to sit quietly while their saliva drains off their lower lip into a collection vessel, known as passive drool, may be employed and such approaches are termed unstimulated methods[18]. Introduction of citric acid to stimulate saliva lowers the $\mathrm{pH}$ which alters the transport properties of some analytes, testosterone for example, and so the most commonly used method for collection of stimulated saliva uses the Salivette [18]. The Salivette is a cotton roll that the participant chews until it is saturated, after which the sample is recovered by centrifugation. The use of cotton wool introduces artefacts into the analysis and has been reported to enhance or reduce the results of immunoassays [18]. A study comparing bacterial levels in unstimulated samples obtained from spitting and passive drool reported 14-fold more bacteria in specimens obtained from spitting than those obtained by passive drool [18]. Bacterial action within a saliva sample is a vital factor operating from the moment sampling starts hence the passive drool approach is commonly adopted to minimise such effects [19].

A Standardised saliva collection method has yet to be realised ,and poor compliance with the many varied interpretations of sampling protocols have been reported [20]. It appears to be accepted that obtaining representative and reproducible whole saliva samples is a nontrivial task. Once collected sample handling and storage procedures are also important with bacteria in the sample continuing to metabolise compounds and degrade the samples' integrity. Many salivary constituents have a short biological half-life, and rapidly degrade after sample collection. Further, the more volatile components are likely to be lost to the 
headspace or adsorbed/absorbed onto the surfaces of the materials used during collection and storage (cotton wool for instance). Storage procedures specify freezing, or cooling, saliva samples to stabilise them and inhibit bacterial degradation action, often neglect volatility. One review of the salivary specimen as a new tool for investigation recommends aliquoting immediately on collection followed by storage at $4^{\circ} \mathrm{C},-20^{\circ} \mathrm{C}$ or $-80^{\circ} \mathrm{C}$ depending on the proposed storage time [18]. Volatility was not a factor in such discussions. Other sample stabilisation steps include inhibition of enzyme or bacterial activity by snap freezing, or addition of inhibitors, denaturing agents or sodium azide for example [2 and 18]. Such measures are time consuming, increase the number of steps in the analytical pathway, and are susceptible to human error, while ensuring significant ventilation of the sample's headspace with the attendant loss of volatiles. The resultant sampled material may well not be truly representative of the salivary composition at the moment of sampling [18]. The challenges associated with reproducible collection and storage of saliva samples as well as the plethora of pre-treatment techniques necessary for a range of analysis techniques have been reviewed elsewhere [1, 2, 16, 17, 18, 21, 22 and 23$]$.

Many of these challenges have been addressed by adopting solid phase microextraction (SPME), for SPME offers simplicity, speed, reliability, and flexibility. Time-consuming preconcentration is reproducibly achieved during sampling, and SPME integrates easily with GC instrumentation. Although SPME offers many improvements over established saliva sampling techniques for volatile components the challenges associated with standardisation of collection, stabilisation and storage of drooled saliva still exist.

This study adopts a polydimethylsiloxane (PDMS) based sampling approach, previously described for sampling VOC from human skin [24] to in-vivo sampling of saliva within the mouth for VOC. This approach removed the pre-treatment, collection and handling complications associated with drooled/expressed saliva methods. This new approach was straightforward to use and compliance with the sample protocol was achieved more reliably. With sampling times between 1 and 30 minutes, "rich" and complex gas chromatographymass spectrometry (GC-MS) VOC profiles may be obtained with up to 600 resolved and partially resolved components. 


\section{EXPERIMENTAL}

\section{Ethics and participant preparation}

The study was conducted in accordance with the ethical principles of Good Clinical Practice and the Declaration of Helsinki. The local ethics committee (Ethical Advisory Committee, Loughborough University, Loughborough, LE11 2DT) approved the studies references G10P23 and G10-P24, and all participants were healthy adult volunteers who gave written informed consent.

Participants were aged 18-35 and were recruited from Loughborough University staff, students and their social networks. A brief introduction and familiarisation with the procedure was given to participants before the beginning of the study and before each sample was taken. Participants were asked not to eat, nor drink anything, other than unflavoured water, brush their teeth or use any personal care products on the morning of their study visit. All samples were taken in a small internal room at the Centre for Analytical Science within the Chemistry Department at Loughborough University. A chaperone, of the same gender as the participant, was present during sample collection and access was restricted to only those researchers and participants involved in the sampling process.

\section{Sampling}

In-vivo sampling.

A saliva sampler, Figure 1 and Table 1, was designed and built that consisted of a solid phase extraction cartridge (Cat no: C-SPTD5-6MM Markes International Ltd) mounted on a threaded pin that screwed securely into a stainless steel holder that could be placed underneath the tongue in an analogous manner to an oral thermometer. The solid phase extraction cartridge cartridges were made from a titanium cylinder coated externally with polydimethylsilicone. Prior to use the cartridges were placed in a thermal desorption tube (Cat no: CO-BXXX-0000 Markes International Ltd) and conditioned with $50 \mathrm{~cm}^{3} \mathrm{~min}^{-1}$ of helium at $190^{\circ} \mathrm{C}$ for $60 \mathrm{~min}$. Once conditioned the Samplers were analysed by thermal desorption gas chromatography mass spectrometry (TD-GC/MS) to ensure that they were free from unacceptable levels of VOC contamination as well as providing a final "polish" prior to use with the study's participants. 
$<$ Table 1 Near here. $>$

$<$ Figure 1 near here $>$

Immediately prior to sampling the saliva sampler was assembled, with care taken not to handle the solid-phase extraction cartridges by using stainless steel tweezers. The sample procedure commenced with the participant drinking $50 \mathrm{~cm}^{3}$ of unflavoured water to refresh their mouth. 1 min later the participant placed the saliva sampler into the sublingual region of the mouth. They could use the handle to stabilise and reposition the saliva sampler if needed, see Figure 1. At the end of sampling the participant removed the saliva sampler by drawing it through their closed lips to remove any excess liquid from the surface. The investigator immediately unscrewed the spindle and placed the solid-phase extraction cartridge into a clean glass thermal desorption tube that was then sealed and stored in an airtight container at $4^{\circ} \mathrm{C}$ until analysis. Samples were not stored any longer than $24 \mathrm{hr}$ before analysis. The samples were removed from storage immediately prior to analysis.

\section{Passive Drool.}

Whole saliva samples for comparative studies using a passive drool approach were collected. The participant was seated with their head tilted forward to cause saliva to pool in the front of their mouth. The saliva was allowed to flow from their mouth into a glass collection vessel fitted with a screw top cap. This process was repeated until approximately $3 \mathrm{~cm}^{3}$ of saliva had been collected. $1.8 \mathrm{~cm}^{3}$ was immediately transferred to a $2 \mathrm{~cm}^{3}$ vial and a solid-phase extraction cartridge fitted to the stainless steel spindle was added rapidly before the vial was sealed. It was important that these procedures were undertaken as quickly as possible to minimise headspace losses. At the end of the extraction time the solid-phase extraction cartridge was removed and quickly and gently wiped with a lint-free wipe ( 'Kimcare' Kimberly-Clark Professional) to remove excess fluids and then placed immediately into a thermal desorption tubes for that was promptly sealed and stored at $4^{\circ} \mathrm{C}$ before analysis within $24 \mathrm{hr}$.

\section{Instrumentation}

VOC were recovered from the sampler with a two-stage thermal desorption procedure (Markes International Ltd Unity2 Thermal Desorber), isolated with a 60m long capillary 
column with a 5\% phenyl, 95\% methyl polysiloxane stationary phase (Cat no: 122-5562 Agilent DB-5MS) and detected using a quadrupole mass spectrometer (Fisons Trio 1000). Table 2 summarises the instrumental parameters.

$<$ Table 2 near here $>$

\section{SAMPLER CHARACTERISATION STUDIES}

Three studies were undertaken to evaluate this approach to characterising the VOC present in human saliva: reproducibility, the effect of sampling time and an evaluation against passive drool-based approaches.

\section{Reproducibility}

The reproducibility of the technique was assessed both in-vitro and in-vivo.

In-vitro

An aqueous $1 \mathrm{ppm}(\mathrm{v} / \mathrm{v})$ standard of five probe compounds, octane, heptanal, methyl hexanoate, nonanol and 2-decanone, representing classes of compound previously observed in human saliva [25] and spanning a representative range of chemical functionality was prepared. $1.8 \mathrm{~cm}^{3}$ of this standard was placed into a $2 \mathrm{~cm}^{3}$ headspace GC sample vial to minimise the headspace whilst allowing for liquid displacement during sampling with a solid-phase extraction cartridge mounted on the stainless steel spindle. The vial was sealed and left to stand in a thermostatically controlled oven at $37^{\circ} \mathrm{C}$ for a sample time of $5 \mathrm{~min}$. At the end of the $5 \mathrm{~min}$ sample time the solid-phase extraction cartridge was removed and quickly and gently wiped with a lint-free wipe to remove excess water and then placed immediately into a thermal desorption tubes for analysis. This procedure was repeated a further five times with a fresh aliquot of standard for each replicate.

In-vivo

Six samples were taken from the sub-lingual region of the mouth of a female participant using the procedure described above with a sampling-time of $5 \mathrm{~min}$. The participant was allowed to rest for $1 \mathrm{~min}$ between each sample. 


\section{In-vivo sampling time}

Six samples taken with sample times $1 \mathrm{~min}, 3 \mathrm{~min}, 5 \mathrm{~min}, 10 \mathrm{~min}, 20 \mathrm{~min}$ and $30 \mathrm{~min}$ were taken from the sub-lingual region of the mouth of a female participant following the procedures described above. The order of sampling was randomised.

Comparative evaluation, in-situ vs. extraction from “passive-drool” samples.

A 10 participant, (5 male and 5 female) double cross-over design was adopted. Each participant provided samples during two separate study visits when they gave in-situ and passive drool samples, see Table 3. Samples were taken before 09:00 hr. on the day of the study visit following the protocols described above. Saliva samples obtained by in-situ sampling and passive drool were extracted for $10 \mathrm{~min}$.

\section{RESULTS AND DISCUSSION}

None of the participants reported any problems arising from using the saliva sampler. No irritation or discomfort was noted and the procedure was well-tolerated.

\section{Reproducibility}

The results from the in-vitro reproducibility study are summarised in Table 4 and Figure 2. The underlying processes controlling the recovery of the probe molecules into the PDMS phase include adsorption followed by diffusion and permeation and these will be a function of concentration, volatility, hydrophobicity and functionality/polarity. The most important predictive molecular characteristic appears to be vapour pressure at $25^{\circ} \mathrm{C}$. As the vapour pressure increases so does the RSD. The amount of material recovered is also inversely correlated to the vapour pressure with the size of the chromatographic response increasing logarithmically with reciprocal vapour pressure, see Figure 3 . Such trends indicate that the most volatile compounds are highly susceptible to small variations in sampling procedures, most rapidly lost from the experiment and recovered with the lowest efficiency; a similar observation has been noted previously [22].

$<$ Table 3 and Figures 2 and 3 near here $>$ 
Figure 4 illustrates the complexity of the chromatography obtained from in-vivo samples with approximately 100 resolved and many more unresolved chromatographic peaks evident. Peak intensities ranging between two to three orders of magnitude.

Eight randomised peaks selected from the study were assessed for normal and lognormal distributions using the Anderson-Darling test [26],and the cumulative distribution function appeared to fit a lognormal distribution more closely than a normal one. Indeed log-normal distributions are often observed in biological systems; cell division of bacteria, latent periods of infectious diseases, permeability and solute mobility in plant cuticles for example as well as antibody concentration in human blood sera [27 and 28]. The "S"-shaped curves, linear probability plots, and symmetric distribution expected in normally distributed data were not observed, Figure 5 provides an example of this behaviour.

For the in-vivo reproducibility data six compounds were selected at random across the chromatogram and where appropriate identified from NIST mass-spectral matches: propanoic acid, an unassigned entity, 2-phenoxy ethanol, n-hexyl salicylate, benzoic acid ester-1, and finally another benzoic acid ester. For these probe compounds the hypothesis of normality was rejected and a log-normal distribution was adopted on the basis that, no significant departure from a log-normal distribution was observed for any them. Figure 5 illustrates this behaviour for the unassigned alkybenzoate (benzoic acid ester-1). Such observations lead to the inference that the levels of VOC in human saliva may follow a lognormal distribution, see Table 5 and Figures 4 and 5.

The data from the in-vitro and in-vivo studies were consistent with a methodology that performed in a predictable and reliable way. Importantly these observations highlighted the priority of sample management in the analytical work-flow, especially for the most volatile components within saliva samples, with recovery and reproducibility strongly influenced by the volatility (expressed as vapour pressure) of the prospective analyte.

\section{In-vivo sampling time}

Figure 6 is a histogram that presents the chromatographic peaks for ethanoic acid, 6methyl-5-hepten-2-one, heptadecane and, an unidentified alkylbenzoate against sampling time. The nature of the chromatography for different elements of the chromatogram is 
discernable along with the degree of enhancement that is possible by increasing sampling time. It is evident that increasing sampling time beyond a limit does not result in increased recoveries of the $\mathrm{VOC}$, and the competitive equilibration processes may be discerned.

Ethanoic acid, was accumulated rapidly over the first $5 \mathrm{~min}$ of sampling. After this the rate slowed reaching a maximum between 5 and $10 \mathrm{~min}$. A further increase in sampling time resulted in a reduced response. Methyl-5-hepten-2-one showed a similar trend of an accumulation to a maximum value followed by a reduction in recovery; in this case the maximum recovery was achieved with a sampling time of ca. $15 \mathrm{~min}$. The less volatile heptadecane and the unidentified alkylbenzoate, present at significantly lower levels, were accumulated with a gradual increase in abundance over $10 \mathrm{~min}$ before tending to equilibrate with the sampler.

The underlying physical chemistry and metabolic/catabolic processes governing these behaviours are likely to involve a combination of: partitioning of VOC from the saliva into the PDMS phase of the sampler cartridge, the continual release of metabolites/catabolites into the saliva and the kinetics of permeation and diffusion of the compounds into and out of the PDMS phase. The role and effect of the different factors on the mass-transport function for each of the many compounds in the VOC saliva profile will be different and reflect the functionality of the compound, its volatility and saliva concentration. Initially, VOC in the saliva may be viewed as partitioning into the PDMS driven by concentration gradients, and more volatile species are likely to penetrate further and accumulate more rapidly in the sampling medium (PDMS). The kinetics of less volatile, and perhaps lower concentration, components will be reflected in a slower accumulation. However, the partition coefficients for compounds of this nature are higher and so as sampling progresses the PDMS sampling phase moves closer to equilibrium with the surrounding saliva and competitive adsorption/absorption processes would result in the displacement of the more volatile components; Equation 1 is helpful in visualising such phenomena.

$$
[i]_{\mathrm{PDMS}}=\frac{K_{i}[i]_{\text {saliva }}}{1+K_{i}[i]_{\text {saliva }}+\sum K_{j}[j]_{\text {saliva }}}
$$


The equilibrium concentration of an analyte in the PDMS sampling phase ([i $\left.]_{\text {PDMS }}\right)$ may be expressed in terms of the partition constant of the analyte between saliva and PDMS $\left(K_{i}\right)$, the concentration of the analyte in saliva $\left([i]_{\text {saliva }}\right)$, and the sum of the products of the concentrations and equilibria constants of the competing co-absorbed species $\left(\sum K_{j}[j]_{\text {saliva }}\right)$.

A second partitioning process also needs to be considered and that is volatilisation into the gas phase from the PDMS phase. This will start as soon as the sampler is removed from the saliva. Fast transfer to the thermal desorption tube is important to minimise evaporative losses and a similar rapid transfer during the thermal desorption procedure is important too. The trends in Figure 3 and Figure 6 reflect these processes, and the sensitivity of volatile components to small changes in sampling processes as already been noted above.

The approximate trends between sampling time and recovery (solid lines in Figure 6) illustrate the point that the optimal conditions for sampling will be different for each analyte and the challenge for profiling VOC in saliva is to develop a method that provides an accurate representation of as many compounds within the target range of the profile as possible. Conversely, this also highlights the inherent selectivity of such techniques and the opportunity to 'tune' sampling methodologies for targeted analysis.

Figure 6 indicates that $10 \mathrm{~min}$ appears to be an acceptable operational compromise for a sampling time and this was the sampling time used for the rest of this study.

\section{Comparison with passive drool}

Figure 7 shows that the chromatography obtained from the two sampling techniques was fundamentally different. While there are many qualitative similarities, with around $70 \%$ of components present in both cases, the differences in intensity were marked. This was especially the case at higher retention times.

The higher recoveries from the in-situ sampler may be the result of contact between the sampler and the surface membranes of the mouth, or the degradation of these compounds once expressed from the mouth. Headspace losses and bacterial degradation may reasonably be anticipated with passively drooled samples. The comparative study was 
definitive in establishing that VOC recoveries were for the most part greater from in-situ sampling.

\section{CONCLUSION}

The reduction of puncture wounds for blood sampling is an end goal of this research, for the increasing prevalence of antibiotic resistant infectious pathogens encourages the development of new diagnostic approaches that are intrinsically non-invasive. The sampling technique described in this study was well-tolerated by our volunteers. Nevertheless a sample time of $10 \mathrm{~min}$ is likely too long to be practical for many envisaged situations. It may be possible to reduce the sampling time to $1 \mathrm{~min}$ with changes in the sampling cartridge construction and the materials used to make it from. What is clear is that this is a simple, reproducible fast and easy to administer approach that may have applications in many diagnostic and metabonomic /metabolomic studies.

Another important element of this research was to develop an analytical work-flow for saliva that was compatible with other in-vivo VOC sampling and analysis methods; breath and skin for instance [22, 29]. Long-term storage stability of samples has not been addressed in this work and will be the subject of future studies. Approaches are likely to include quench freezing in liquid nitrogen or fast re-trapping onto sorbent beds.

In adopting this methodology care needs to be taken to ensure sampling times are carefully controlled and if possible matched to the volatility of the analytes under study. Enhanced sensitivity and selectivity may be achieved by careful optimisation. Conversely, it is possible to introduce bias if this factor is not carefully managed.

The tentative observation of log-normal distributions is potentially important and may have wider ramifications with other in-vivo VOC measurement techniques. A follow up study with this as the main focus would be a logical next step int his regard.

Comparison of in-situ sampling with passive drool collection has revealed that in-situ sampling provides more analytical information with lower intrinsic variability compared to a passive drool approach. The responses in this study are encouraging and indicate significant analytical utility for this form of in-situ sampling. There is sufficient sensitivity and precision 
to enable these data to be included with existing breath and skin analysis methods in VOC profiling and biomarker prospecting studies.

\section{ACKNOWLDGEMENTS}

The authors gratefully acknowledge Unilever PLC's support to Helen Martin. They also want to express their appreciation to Mr Peter Carroll (Unilever Port Sunlight) who built the in-situ sample holder for the SPME cartridges and thank the volunteers who participated in this research.

\section{REFERENCES}

1. S. P. Humphrey and R. T. Williamson, J. Prost. Dent., 2001, 85, 162.

2. R. G. Schipper, E. Silletti and M. H. Vingerhoeds. Arch. Oral Bio.2007, 52, 1114.

3. V. S. Wang and M. Y. Lu, J. Chrom. B. 2009, 877, 24.

4. M. Ferrari, S. Negri, P. Zadra, S. Ghittori and M. Imbriani, Int. Arch. Occup. Envrion. Health. 2008, 81, 1021.

5. E. Kaufman and I. R. Lamster, Crit. Rev. Oral Biol. Med. 2002, 13, 197.

6. M. J. Silva, J. A. Rediy, E. Samandar, A. R. Herbert, L. L. Needham and A. M. Calafat, Arch. Toxicol. 2005, 79, 647.

7. M. Esteban and A. Castaño, Env. Int. 2009, 35, 438.

8. S. Yamada, K. Yamauchi, J. Yajima, S. Hisadomi, H. Maeda, K. Toyomasu and M. Tanaka, Psychiatry Research, 2000, 93, 217.

9. Y. Guan, Q. Chu and J. Ye, Anal. Bioanal. Chem. 2004, 380, 913.

10. Y. Guan, T. Wu and J. Ye, J. Chrom. B. 2005, 821, 229.

11. L. Jollin, R. Thomasson, B. Le Panse, A. Daillot, N. Vibarel-Rebot, A. M. Lecoq, V. Amiot, J. De Ceaurriz and K. Collomp, Eur. J. Clin. Invest. 2010, 40, 183.

12. D. Luo, F. Chen, K. Xiao and Y. Q. Feng, Talanta, 2009, 77, 1701.

13. E. Cognard, S. Bouchonnet and C. Staub, J. Pharma. Biomed. Anal. 2006, 41, 925.

14. A. B. Actis, N. R. Perovic, D. Defago, C. Beccacece and A. R. Eynard, Arch. Oral Bio. 2005, 50, 1.

15. M. Fenske, Chromatgraphia, 2007, 65, 233.

16. H. W. A. Teeuwen, E. L. Elbers and J. M van Rossum, Molecular Biology Reports, 1991, 15, 1. 
17. J. K. M. Aps and L. C. Martens, Forensic Sci. Int. 2005, 150, 119.

18. S. Chiappin, G. Antonelli, R. Gatti and E. F. De Palo, Clinica Chimica Acta, 2007, 383, 30.

19. Salimetrics, "Saliva Collection and Handling Advice", Publ. Salimetrics Europe, Newmarket, CB8 7SY, UK. 2009. http://www.salimetrics.com/literature/ , downloaded 30 March 2012.

20. D. A. Granger, K. T. Kivlighan, C. Fortunato, A. G. Harmon, L. C. Hibel, E. B. Schwartz and G. Whembolua, Physiol. Behav. 2007, 92, 583

21 M. W. J. Dodds, D. A. Johnson and C. Yeh, J. Dentistry, 2005, 33, 223

22. D. Malamud, Dent. Clin. N. Am. 2011, 55, 159

23. A. Lochner, L. Weisner and A. Zlatkis, J. Chrom, 1986, 378, 267.

24. S. Riazanskaia, G. Blackburn, M. Harker, D. Taylor and C. L. P. Thomas, Analyst. 2008, 133, 1020.

25. H. Soini, I. Klouckova, D. Wiesler, E. Ouberzaucher, K. Grammer, S. J. Dixon, Y. Xu, R. G. Brereton, D. J. Penn and M. V. Novotny, J. Chem. Ecol, 2010, 36, 1035.

26. M. A. Stephens, J. Am. Statistical Assoc., 1974, 69, 730.

27. J. N. Miller and J. C. Miller, "Statistics and Chemometrics for Analytical Chemistry" 5th Edition, Pearson Education Ltd., 2005.

28. E. Limpert, W. A. Stahel and M. Abbt, Bioscience, 2001, 51, 341.

29. M. Basanta, T. Koimtzis, D. Singh, I, Wilson and C. L. P. Thomas. Analyst. 2007, 132, 153. 


\section{SAMPLING AND CHARACTERISATION OF VOLATILE ORGANIC COMPOUND PROFILES IIN HUMAN SALIVA USING A POLYDIMETHYLSILOXANE COUPON PLACED WITHIN THE ORAL CAVITY.}

\section{FIGURES}

Helen J Martin ${ }^{1}$, Svetlana Raisanskaia, ${ }^{2},{ }^{1}$ C. L. Paul Thomas ${ }^{1}$. Centre for Analytical Science, Department of Chemistry, Loughborough University, Loughborough, Leicestershire. LE11 3TU. UK 


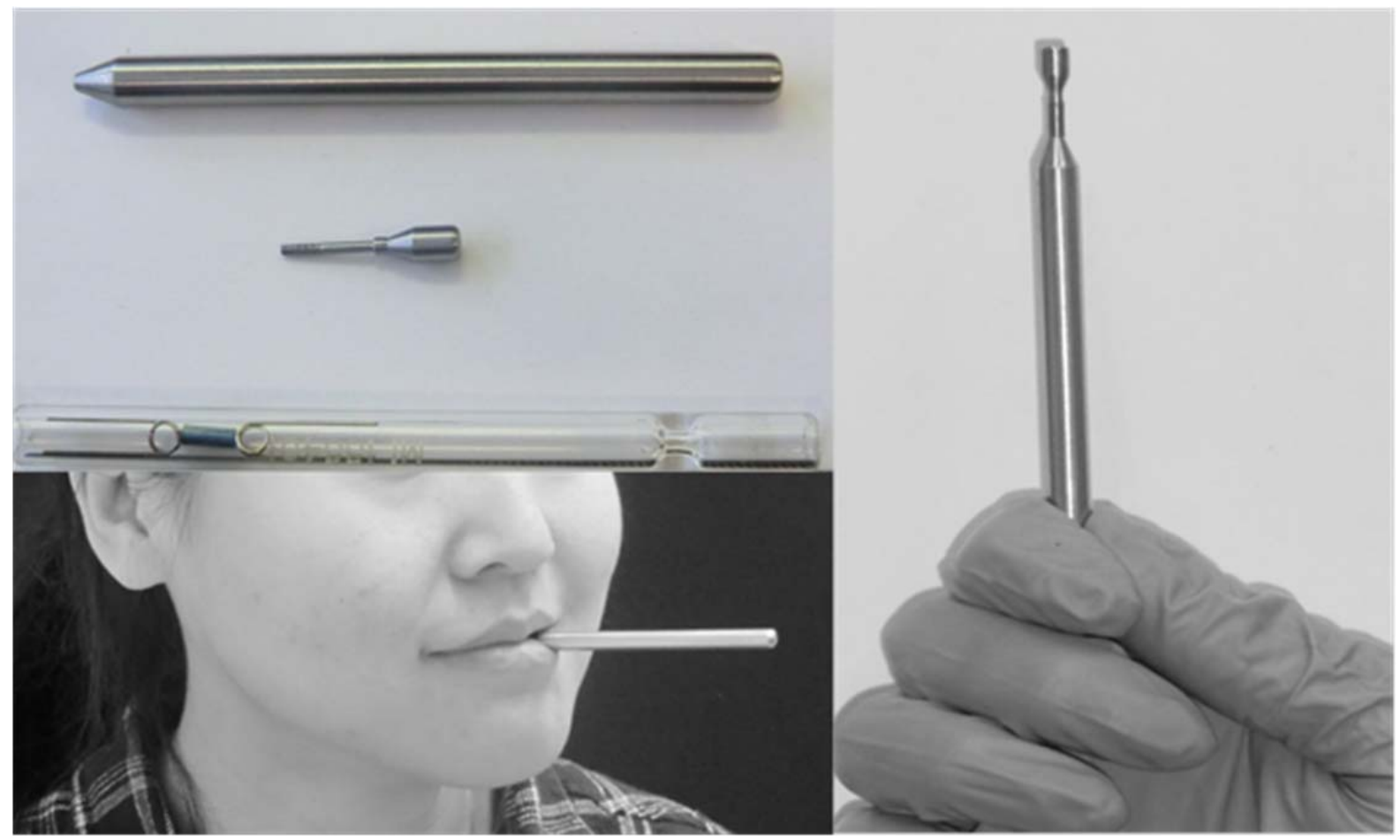

Figure 1. Saliva sampler. Clockwise from top left. A titanium cylinder coated with polydimethylsiloxane is held in place by two springs inside a glass thermal desorption tube. Once conditioned the cylinder was removed and placed onto the threaded stainless steel spindle that was then screwed into the holder, (Right). The assembled sampler could then be placed comfortably into the mouth of a participant where a VOC sample was collected in-vivo (Bottom left). 


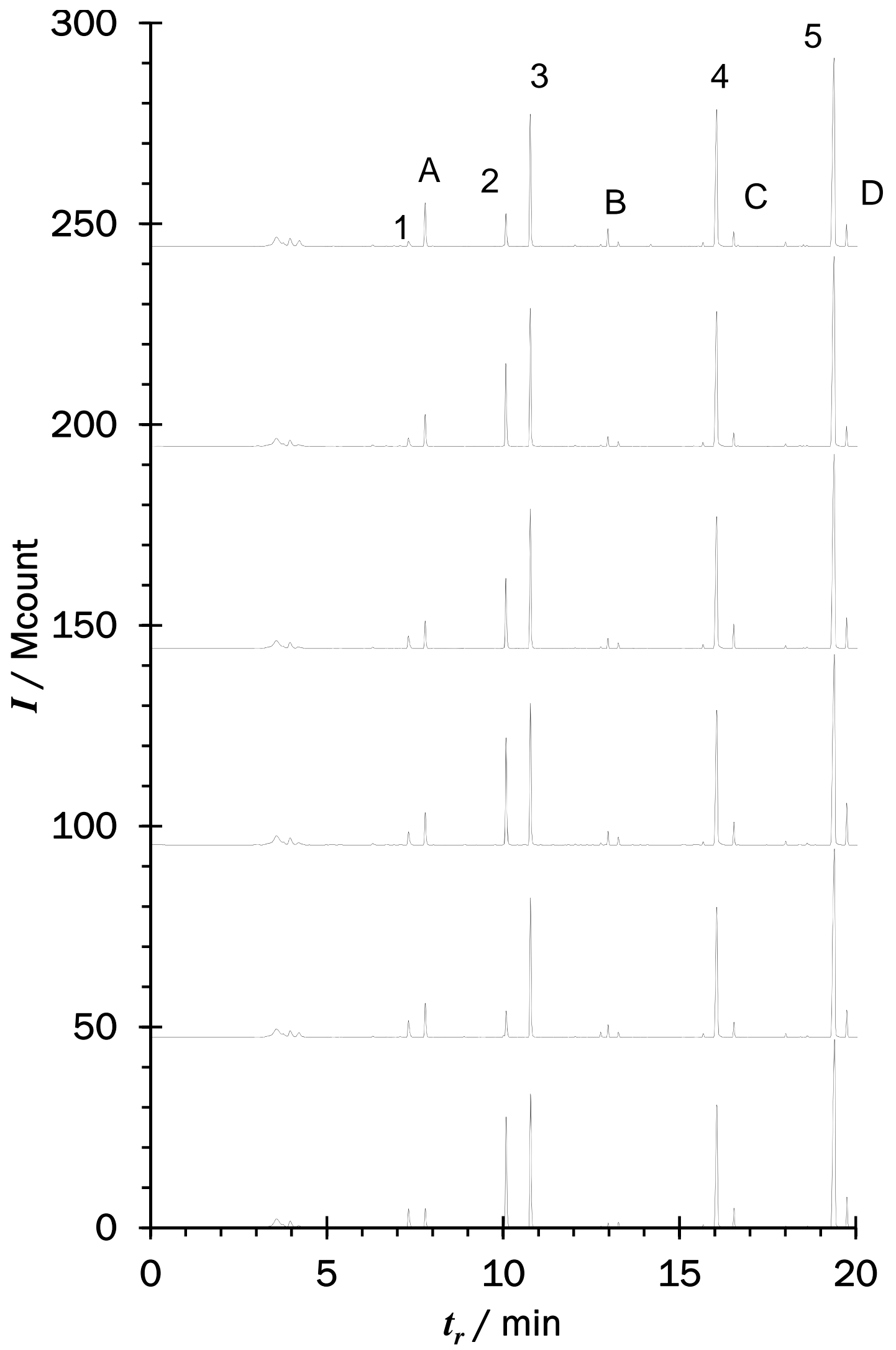

Figure 2. The six replicates from the in-vitro reproducibility study. Six replicate samples of a $1.8 \mathrm{ml}$ aliquot of an aqueous standard containing $1 \mathrm{ppm}$ octane (1), heptanal (2), methyl-heanoate (3), 5-nonanol (4) and 2-decanone (5). Also labelled are compounds from the PDMS sampling medium (A-D). 


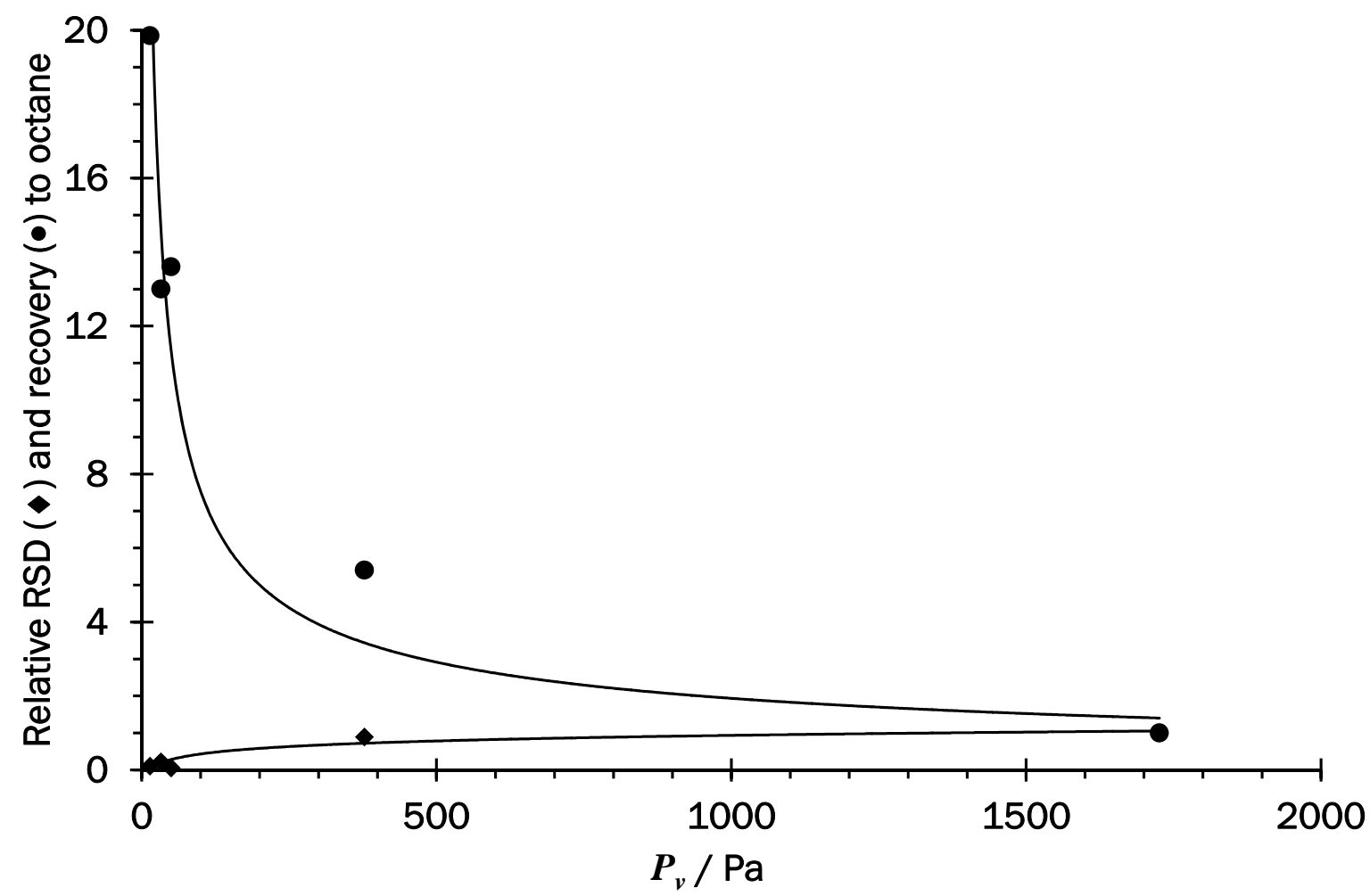

Figure 3. Examination of the recovery data (circles) and reproducibility data (diamonds)in Table 4 from the in-vitro reproducibility reveals how vapour pressure predicts the recovery and reproducibility of VOC. These two plots show how RSD increases, and recovery reduces, with vapour pressure. These data are plotted relative to those of octane to enable both trends to be viewed easily on the same graph.

Data for octane (Tabl e 4), $P_{v}: 1726 \mathrm{~Pa}$ at $25^{\circ} \mathrm{C}, A: 20$ kcount s, and RSD: $26 \%$. 


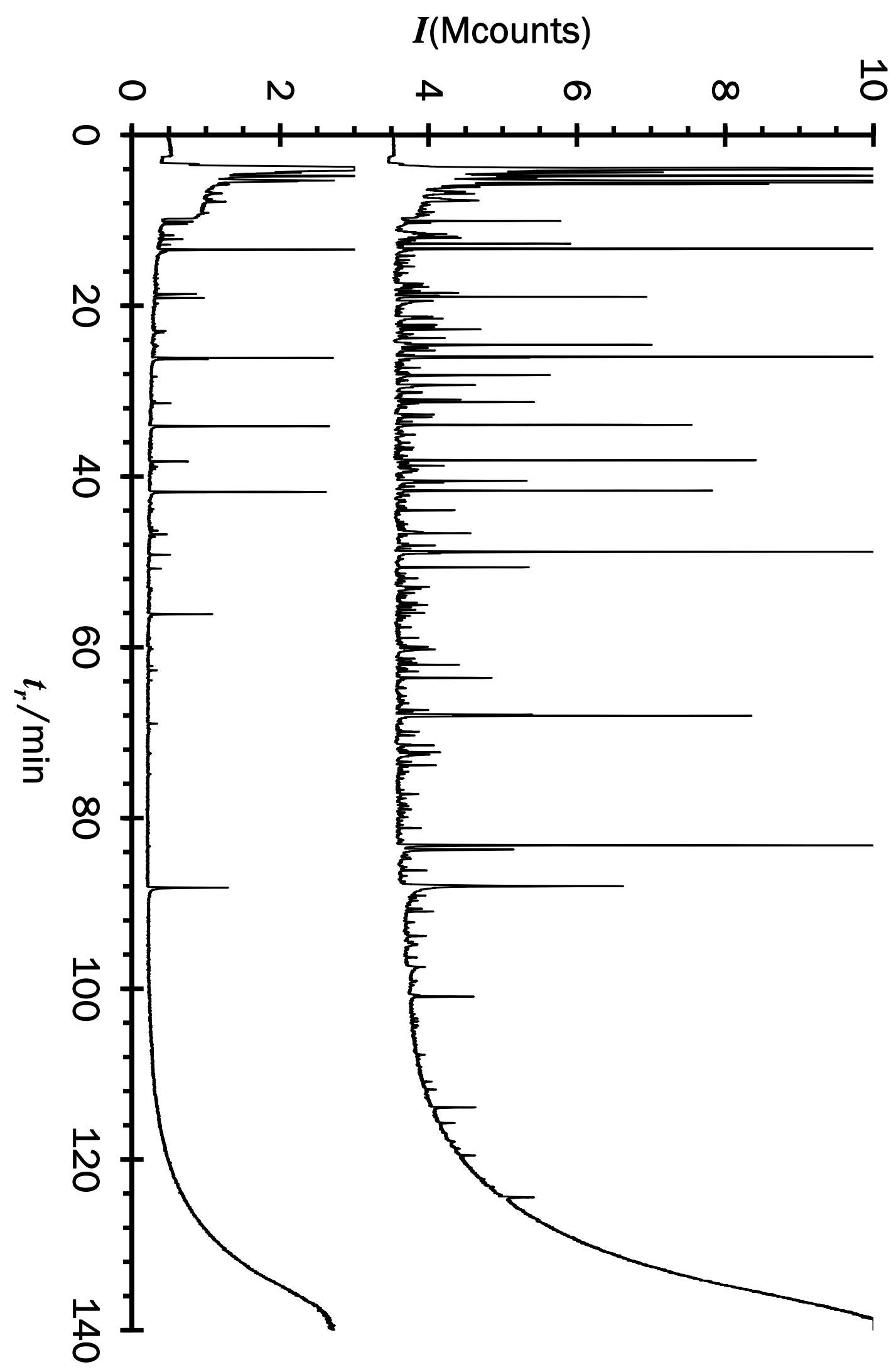

Figure 4. Top: An example chromatogram of a VOC in saliva profile obtained from in-vivo sampling with a polydimethylsilicone cartridge sampler, (Tables 1 and 2). Bottom: The corresponding response from a sampler blank. Traces are magnified $\times 5$ with a 3 MCounts cut of for the blank and a 10 MCounts cut-off for the saliva trace. 

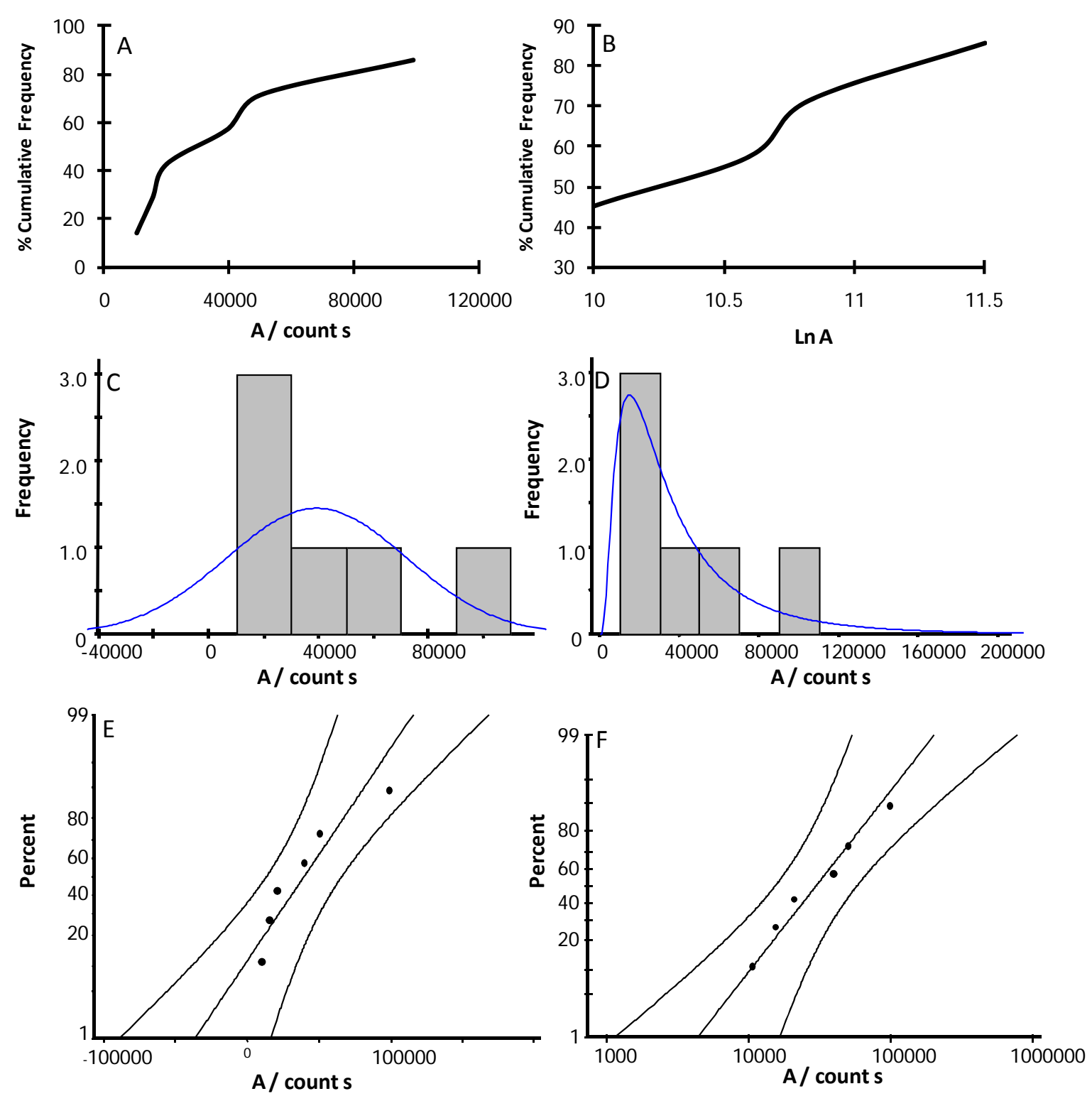

Figure 5. Example of evidence for log-normal distributions of VOC concentrations in human saliva. Cumulative frequency curves for Component 5 in Table 5 a benzoic acid ester $t_{r}=2616 \mathrm{~s}$. Showing normal (A) and log-normal (B) distributions, histograms for normal (C) and lognormal (D) distributions and probability plots for normal $(E)$ and lognormal (F) distributions suggest a lognormal distribution of VOC levels in the participant studied for in-vivo reproducibility.- 


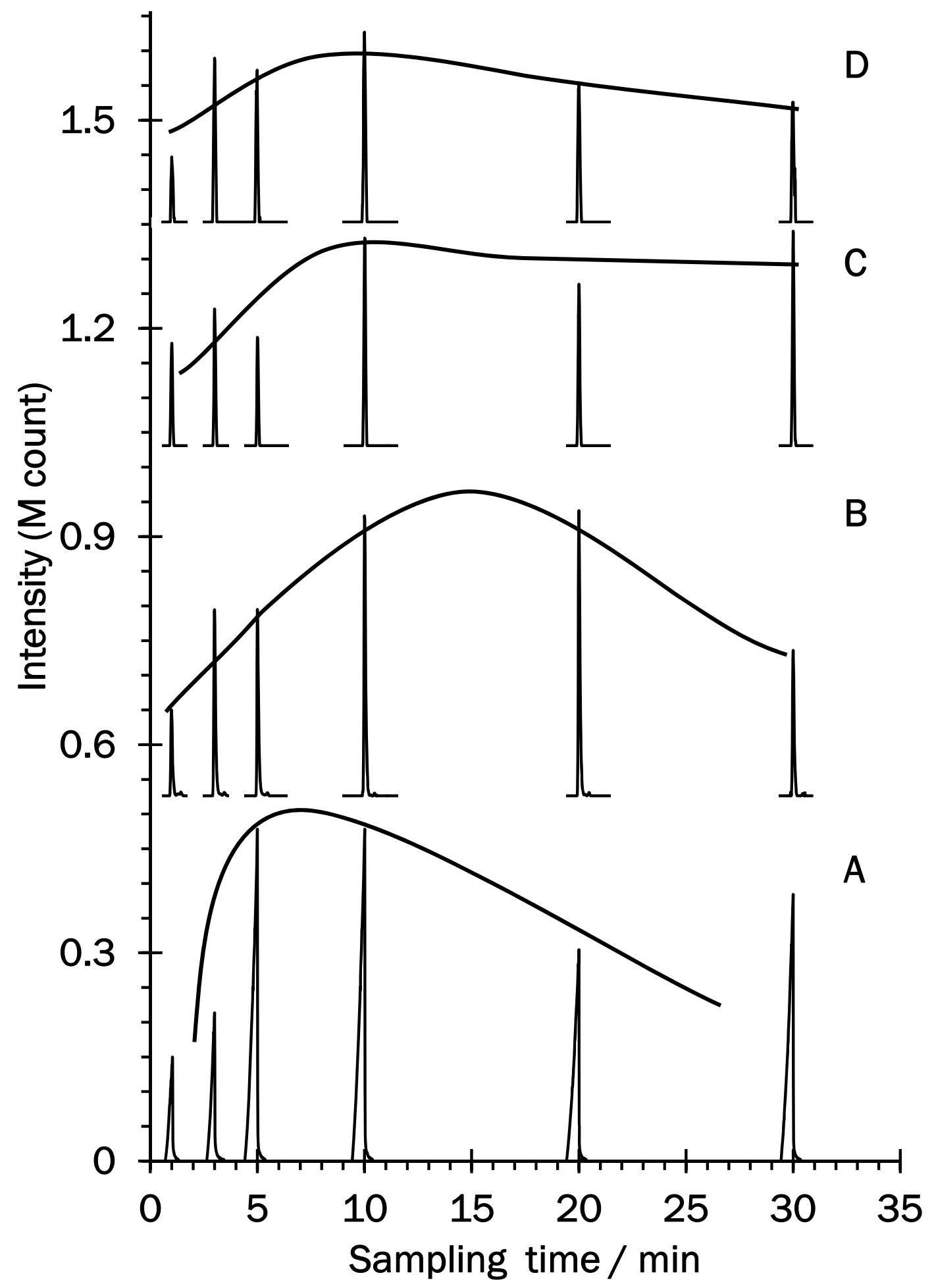

Figure 6. A histogram showing the effect of sampling time on the recovery of: ethanoic acid (A, tr $5.9 \mathrm{~min}$ ); 6-methyl-5-hepten-2-one (B, tr $25.1 \mathrm{~min}$ ); heptadecane at x10 magnification (C, $73.8 \mathrm{~min}$ ); and, an unidentified alkylbenzoate at $\times 100$ magnification (D, $112.6 \mathrm{~min}$ ). The deconvolved peaks for these VOC are shown against different sampling times. The solid lines provide an indication of the general trends of accumulation, and show the combined effects of the partition constants, concentration gradients, and the metabolic/catabolic release into the oral cavity. 

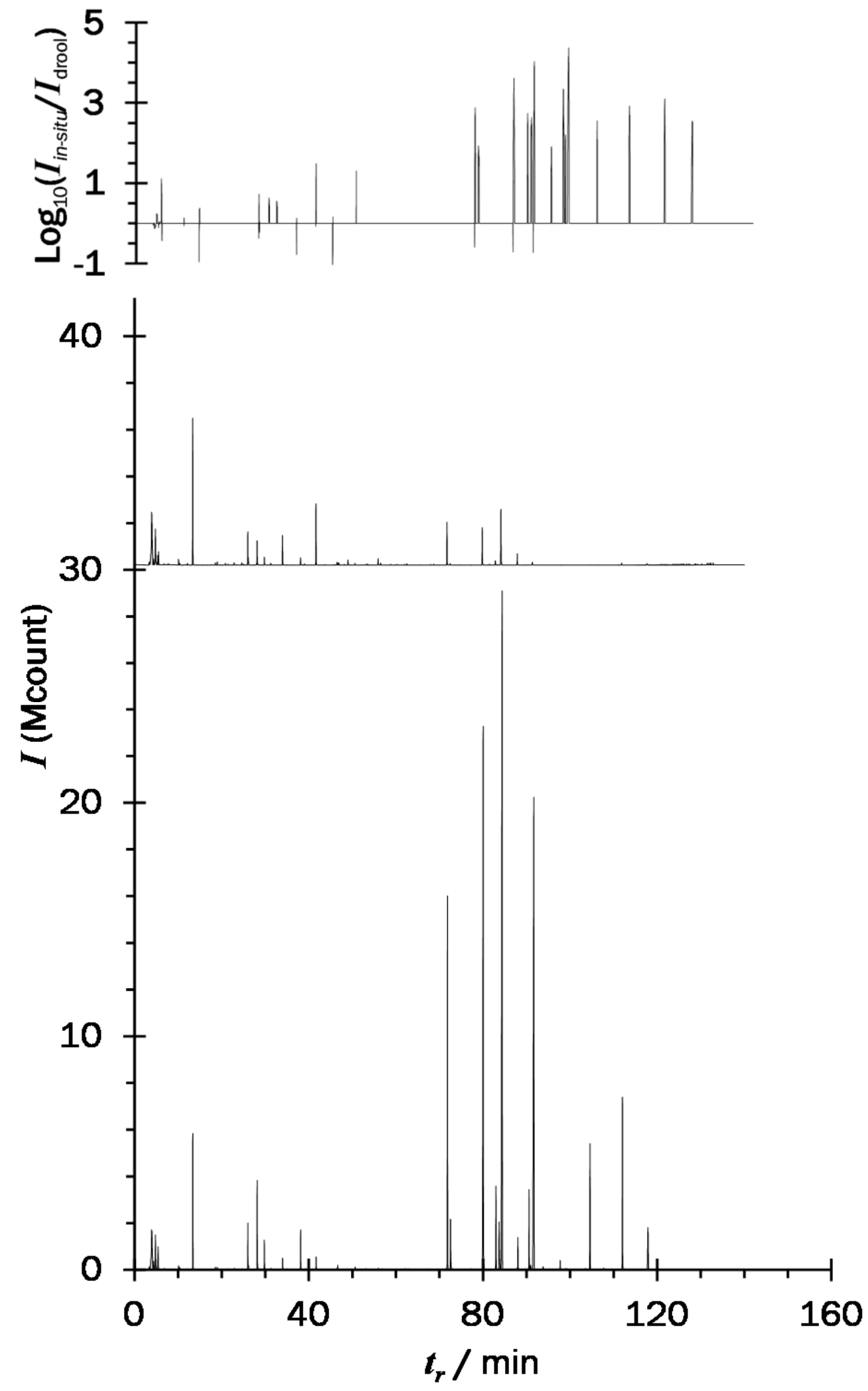

Figure 7. A comparison of the GC profiles observed from a PDMS in-situ sample bottom, and from a passive drool, offset at 30 Mcount. The top trace shows the log of the relative responses $\left(\log _{10}\left(I_{\text {in-situ }} / I_{\text {Drool }}\right)\right.$ for all the peaks with an intensity of greater than $1 \%$ of maximum. The differences in the profiles indicated significant enhancement in signals obtained from the in-situ samples. 


\section{SAMPLING AND CHARACTERISATION OF VOLATILE ORGANIC COMPOUND PROFILES IIN HUMAN SALIVA USING A POLYDIMETHYLSILOXANE COUPON PLACED WITHIN THE ORAL CAVITY.}

TABLES

Helen J Martin ${ }^{1}$, Svetlana Raisanskaia ${ }^{2},{ }^{1}$ C. L. Paul Thomas ${ }^{1}$.

Centre for Analytical Science, Department of Chemistry, Loughborough University, Loughborough, Leicestershire. LE11 3TU. UK

Unilever R\&D, Port Sunlight, Quarry Road East, Bebington, Wirral, UK. .

${ }^{1}$ Corresponding Author,c.l.p.thomas@Iboro.ac.uk 
Table 1. Summary of design parameters for the saliva sampler.

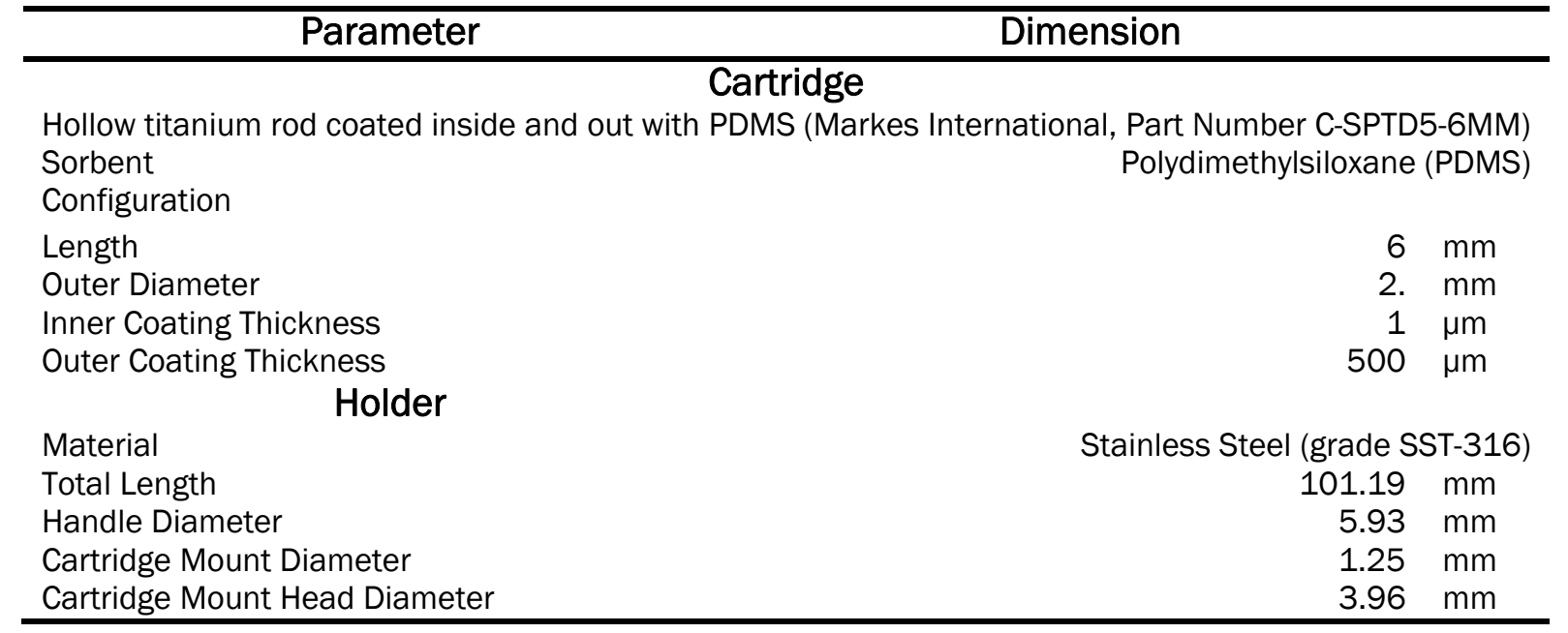


Table 2. Summary of instrumentation operating parameters.

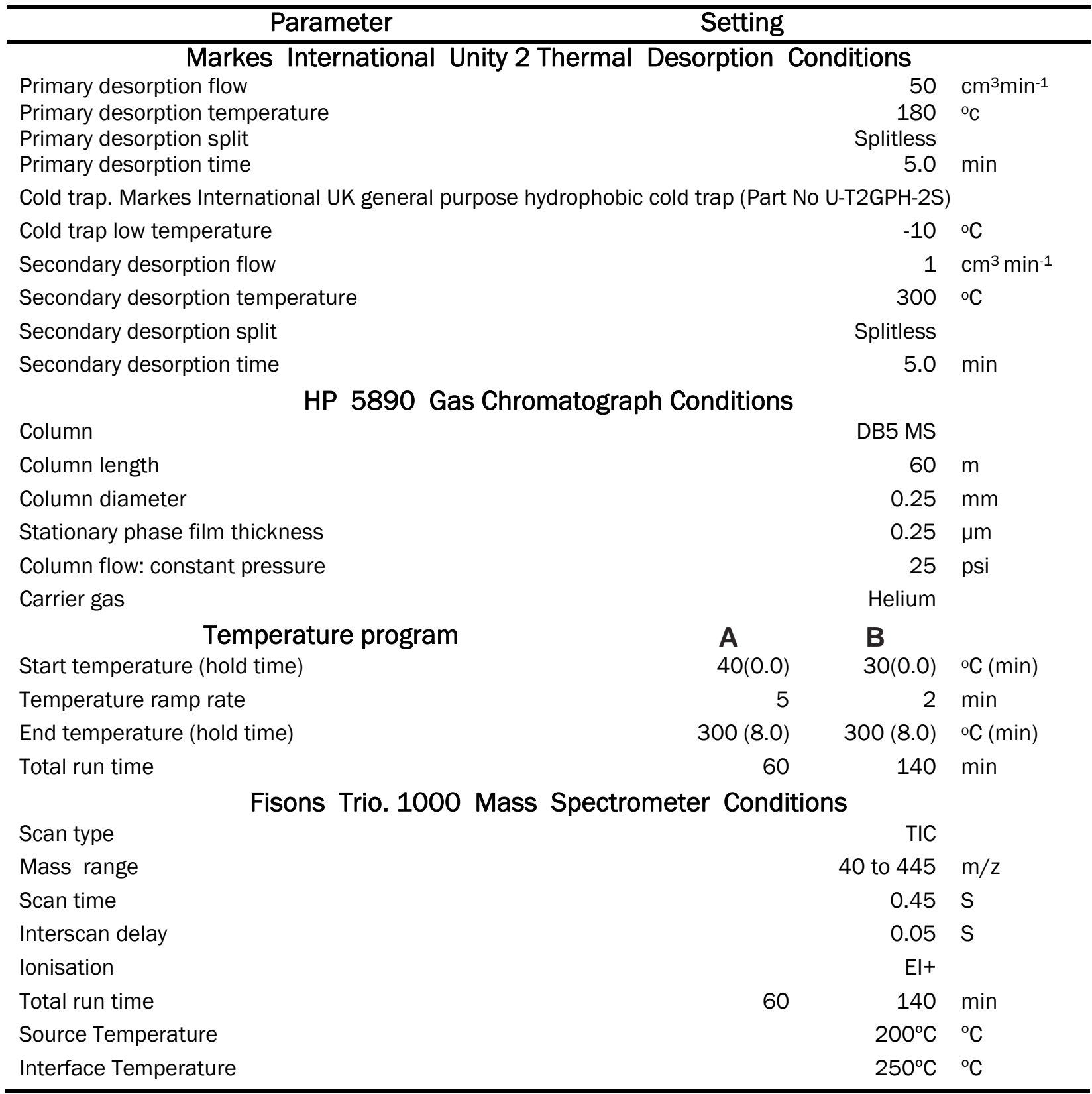

Note. A: Temperature program for reproducibility studies

B:Temperature program for sampling time and comparison-evaluation studies 
Table 3. Summary of the sampling campaign adopted for the in-situ vs. passive drool evaluation study.

\begin{tabular}{|c|c|c|c|c|c|c|c|c|c|c|}
\hline Sample order & F-02 & M-03 & M-04 & M-05 & $\mathrm{M}-06$ & F-01 & F-07 & F-08 & $\mathrm{M}-10$ & F-09 \\
\hline $1-1$ & I-S\#01 & I-S\#03 & P-D\#01 & I-S\#09 & I-S\#13 & P-D\#15 & P-D\#17 & P-D\#19 & P-D\#23 & I-S\#25 \\
\hline $1-2$ & P-D\#02 & P-D\#04 & I-S\#08 & P-D\#10 & $P-D \# 14$ & I-S\#16 & I-S\#18 & I-S\#20 & I-S\#24 & P-D\#26 \\
\hline $2-1$ & P-D\#05 & P-D\#11 & I-S\#33 & P-D\#21 & P-D\#39 & 1-S\#37 & I-S\#31 & I-S\#27 & I-S\#35 & P-D\#29 \\
\hline $2-2$ & I-S\#06 & I-S\#12 & P-D\#34 & I-S\#22 & I-S\#40 & P-D\#38 & P-D\#32 & P-D\#28 & P-D\#36 & I-S\#30 \\
\hline
\end{tabular}

Note: $\quad$ Sample order: $x-y$ where $x$ : visit number and $y$ : sample number. The participant code designates gender (M/F) and their study identifier number. Each sample is designated as I-S: in-situ or P-D passive drool followed by the position in campaign sequence \#. The order of the participants reading left to right designates the order of their recruitment to this study. 
Table 4. Summary of the in-vitro reproducibility study.

\begin{tabular}{llrrrrrrr}
\hline & Compound & $\boldsymbol{M} . \boldsymbol{W t}$. & $\boldsymbol{P}_{\boldsymbol{v}} / \mathrm{Pa}$ & $\boldsymbol{T}_{\boldsymbol{B}} / \mathrm{K}$ & $\mathbf{Q}(\mathrm{m} / \mathbf{z})$ & $\boldsymbol{t}_{\boldsymbol{r}} / \mathbf{s}$ & $\boldsymbol{A} / \mathrm{kcount} \mathbf{s}$ & $\mathrm{RSD}$ \\
\hline 1 & Octane & 114.14 & 1726 & 399 & 43 & 438 & 20 & 26 \\
2 & Heptanal & 114.10 & 378 & 426 & 70 & 606 & 108 & 23 \\
3 & Methyl hexanoate & 130.10 & 50 & 423 & 74 & 648 & 272 & 1.3 \\
4 & 5-Nonanol & 144.15 & 14 & 468 & 69 & 966 & 397 & 2.7 \\
5 & 2-Decanone & 156.15 & 33 & 484 & 58 & 1164 & 260 & 5.5 \\
\hline
\end{tabular}

Note. $\quad M . W t$, relative molecular mass; $\boldsymbol{P}_{\boldsymbol{v}}$, Vapour pressure $/ \mathrm{Pa}$ at $25^{\circ} \mathrm{C}$ (taken from Chemspider, www.chemspider.com); $T_{B}$, Boiling point / $\mathrm{K} ; Q$, Quantitation ion $(\mathrm{m} / \mathrm{z}) ; t_{r}$,retention time / $\mathrm{s} ; A$, chromatographic peak area /kcount s; and, RSD, relative standard deviation (\%). 
Table 5 Comparison of Anderson-Darling statistics for 8 peaks selected at random, from the in-vivo reproducibility study. The Anderson-Darling test [ref] is a goodness of fit test based on the culmulative frequency function. The $A D$ statistic measures how well the data follow a particular distribution, the better the fit the smaller $A D$ value. The $p$-value is the probability of obtaining these test results when the assumed distribution of the data is true. Therefore when comparing distributions to determine the best fit it is generally useful to compare the $A D$ and $p$ values, the distribution with the smaller $A D$ and larger $P$ values best fit the data.

These components appear to follow the lognormal distribution more closely.

\begin{tabular}{lrrrr}
\hline$t_{r} / \mathbf{s}$ & Normal Distribution & \multicolumn{2}{c}{ Lognormal Distribution } \\
& $\mathrm{AD}$ & $\mathbf{p}$ & $\mathrm{AD}$ & $\mathbf{p}$ \\
\hline 1098 & 0.930 & 0.007 & 0.417 & 0.214 \\
1218 & 0.336 & 0.364 & 0.213 & 0.733 \\
1776 & 0.336 & 0.364 & 0.169 & 0.882 \\
1962 & 0.311 & 0.428 & 0.158 & 0.904 \\
2334 & 1.081 & $<0.005$ & 0.300 & 0.459 \\
2400 & 0.740 & 0.025 & 0.256 & 0.570 \\
2616 & 0.422 & 0.207 & 0.169 & 0.884 \\
2724 & 0.740 & 0.025 & 0.256 & 0.570 \\
\hline
\end{tabular}

Table 6 Summary of the in-vivo reproducibility study

\begin{tabular}{llrrrr}
\hline & Assignment & $\mathrm{Q}(\mathrm{m} / \mathrm{z})$ & \multicolumn{1}{c}{$t_{r} / \mathbf{s}$} & \multicolumn{1}{c}{$\ln \boldsymbol{A}$} & \multicolumn{1}{c}{$\mathrm{RSD}$} \\
\hline 1 & Propanoic Acid & 74 & 312 & 7.62 & 18.2 \\
2 & Unassigned & 106 & 1098 & 5.77 & 15.7 \\
3 & 2-phenoxy Ethanol & 94 & 1218 & 4.94 & 6.4 \\
4 & n-Hexyl Salicylate & 120 & 1962 & 6.68 & 9.9 \\
5 & Benzoic acid ester -1 & 123 & 2616 & 10.29 & 8.8 \\
6 & Benzoic acid ester-2 & 123 & 2724 & 10.27 & 9.4 \\
\hline
\end{tabular}

Note. $\quad Q$, Quantitation ion $(\mathrm{m} / \mathrm{z}) ; t_{r}$, retention time / $\mathrm{s} ; A, \quad$ mean chromatographic peak area / kcount s; and, RSD, relative standard deviation (\%). 